Journal of Statistical Physics, Vol. 93, Nos. 1/2, 1998

\title{
On the Unicity of Discontinuous Transitions in the Two-Dimensional Potts and Ashkin-Teller Models
}

\author{
T. Baker ${ }^{1}$ and L. Chayes ${ }^{1}$
}

Received January 9, 1998

We investigate the uniqueness of the discontinuous phase transition of the $q$-state Potts model and the symmetric $q=(s \times s)$ cubic model (generalized Ashkin-Teller model) on the integer square lattice with $q \geqslant 1$. For the ferromagnetic cases, when there is a discontinuous phase transition we show that the discontinuity can only occur at a point of self-duality.

KEY WORDS: Ashkin-Teller model; Potts model; graphical representations; percolation; duality.

\section{INTRODUCTION}

The two-dimensional versions of the Potts and Ashkin-Teller (AT) models enjoy the property of duality which transforms a model at high temperature into one at low temperature. This allows for an educated guess as to the location of points where there are phase transitions: points that are self-dual. Here, for the Potts model, we establish that when there is a discontinuous transition, this transition is unique and is located precisely at the self-dual point. For the AT model, a similar statement is proved under the proviso that all interactions are ferromagnetic.

For the $2 d$ Potts model and its random cluster generalization, it is universally accepted that for $q \leqslant 4$, the transition is continuous and is discontinuous for $q>4$. Only part of this picture has been actually proved: The cases $q=1,2$ are known to be continuous and for integer $q \gg 1$ the transition was proved to be discontinuous [KS] by the method of reflection positivity. The preceding is dimension independent; specific to $d=2$, is

\footnotetext{
${ }^{1}$ Department of Mathematics, University of California, Los Angeles, California 90024; e-mail: tbaker $(a$ ucla.edu; lchayes $a$ math.ucla.edu.
} 
the result of [LMMsRS] where for all $q \gg 1$ it was shown that the transition occurs at the self-dual point (and that the free energy is analytic elsewhere). Subsequently this has been improved to less extreme values of $q$, see, e.g., [A]. The same general trend presumably holds for the $(r \times s)$-AT models although the situation is more complicated since more parameters are involved. In any case, not much has been proved; here, at least for integer $r=s \gg 1$ we will show there is a first order transition.

However, in the core of this paper, we will use a track that is different from all of the above mentioned: we restrict attention to the cases $q \geqslant 1$ and $r=s \geqslant 1$ and simply acknowledge that either a discontinuous transition occurs or it does not. In case it does occur, we prove that the transition point is unique and located at the self-dual point.

We will actually study the graphical representation of the $q$-state Potts models and the $(s \times s)$-state AT-models. These representations, which make sense even for non-integer values of $q$ or $s$, have been shown to faithfully describe the models in the sense that the Gibbs distributions in finite volume with fixed boundary conditions are completely determined by the distributions of the graphical representation ([ACCN] and $[\mathrm{CM}])$. In the regions of interest, the phase structure of these models are characterized by the percolation properties of the graphical representation. In particular, there are multiple Gibbs states in the spin-system if and only if there is percolation in the graphical representation. Furthermore, even for non-integer values of $q$ and $s$, the absence of percolation implies there is a unique state of the random cluster model.

For ease of exposition, we will first do an explicit proof for the Potts cases and then generalize to the AT-cases. (Notwithstanding that the latter contains the former as a special case.) In both cases we show that if there is a discontinuous transition-in the sense of phase coexistence of states with differing bond density (i.e., energy density) then such a point must be a self-dual point. Furthermore, in the high bond density phase, there is percolation (positive spontaneous magnetization) and in the low density phase there is none. Thus, this is the transition point.

Our argument go along the following lines: The transition point for the onset of percolation must have a value greater than or equal to that of the self-dual parameter by well known arguments-namely that the placement of the critical parameter at a point less than the self-dual parameter would, through duality, violate the argument of Gandolfi, Keane, and Russo [GKR] on the uniqueness of the infinite cluster in two-dimensions. However, the (assumed) point of discontinuity in the bond density must be located at a value greater than or equal to that of the critical parameter. This is because a discontinuity in the bond density implies non-uniqueness of the random cluster measure, which then in turn implies the existence of 
percolation. However, if a discontinuity in the bond density were to occur at a parameter value strictly greater than that of the self-dual parameter, duality would place a discontinuity of the vacant bond density at a value less than the self-dual parameter. Since this would imply percolation below the self-dual point (which is impossible) it follows that the discontinuity occurred at the self dual point which must also be the transition point for the onset of percolation.

\section{THE POTTS MODEL}

Let $\Lambda$ denote a finite connected subset of $\mathbb{Z}^{2}: A \Subset \mathbb{Z}^{2}$. Let $\mathbb{S}_{A}$ denote the sites and $\mathbb{B}_{A}$ the bonds of $A$. The $q$-state Potts model is defined by the Hamiltonian

$$
H\left(\sigma_{A}\right)=-\sum_{\langle i, j\rangle \in \mathbb{B}_{\Lambda}} \delta_{\sigma_{i} \sigma_{j}}
$$

where $\left(\sigma_{i} \mid i \in \mathbb{S}_{A}\right)$ are the "spins" taking values $\sigma_{i} \in\{1,2, \ldots, q\}, \delta_{\sigma_{i} \sigma_{j}}$ is the usual Kronecker-delta and $\sigma_{A}$ is a spin configuration: $\sigma_{A} \in\{1,2, \ldots, q\} \mathbb{S}_{A}$. The partition function, at inverse temperature $\beta$ is given by

$$
Z_{A, \beta}=\sum_{\sigma_{A}} e^{-\beta H\left(\sigma_{A}\right)}
$$

where so far (and in future as much as possible) we have neglected a discussion of the boundary conditions. Via the FK random cluster representation $[\mathrm{FK}]$ the partition function is given by the expression

$$
Z_{A, \beta}=\sum_{\omega} V(\omega)
$$

where $\omega \in\{0,1\}^{\mathbb{B}_{A}}$ is a configuration of occupied $\left(\omega_{\langle i, j\rangle}=1\right)$ and vacant $\left(\omega_{\langle i, j\rangle}=0\right)$ bonds. The quantity $V(\omega)$ is given by

$$
V(\omega)=R^{|\omega|} q^{c(\omega)}
$$

with $R=e^{\beta}-1,|\omega|$ the number of occupied bonds and $c(\omega)$ the number of connected components. Since for each $\omega$, the quantity $V(\omega)$ is positive, these weights define a probability measure which we will denote by $\mu_{F K ; A}^{q, R}(-)$. Here $q$ need not be an integer.

A great deal is known about these measures, their finite-volume properties, and the infinite volume limits thereof. Much of this was derived in $[\mathrm{ACCN}]$, see also $[\mathrm{CM}]$ and the (invited!) review article $[\mathrm{G}]$. Of 
relevance here are the FKG properties and the connection between percolation and phase transitions.

We must discuss, a bit, the question of boundary conditions. In finite volume, for all formulas as written, we have the free boundary condition measures which, in fact, correspond to free boundary conditions in the spin system. Alternatively, we may consider all sites that are connected to the boundary as part of the same cluster and this interpretation of the symbol $c(\omega)$ defines the wired measures. (This, back in the spin-system, amounts to setting all the boundary spins to the same value). We will denote the influence of boundary conditions on our measures by a superscript on the $A$. E.g., $\mu_{F K ; A^{\prime \prime}}^{q, R}(-)$ is the wired measure.

For $q \geqslant 1$ (which henceforth will always be assumed) both the free and wired measures enjoy the FKG property, that is to say there are positive correlations between events that are increasing with respect to the natural partial order on bond configurations. A measure $\mu_{1}(-)$ is said to FKG dominate another measure, $\mu_{2}(-)$ (defined on the same space) if, for every increasing event $\mathscr{A}$ we have $\mu_{1}(\mathscr{A}) \geqslant \mu_{2}(\mathscr{A})$. Such a relation will be denoted by $\mu_{1}(-) \geqslant_{\mathrm{FKG}} \mu_{2}(-)$. For our purposes, it should be noted that if $R_{1}>R_{2}$, then $\mu_{F K ; A^{*}}^{q, R_{1}}(-) \geqslant_{\mathrm{FKG}} \mu_{F K ; A^{*}}^{q_{1}, R_{2}}(-)$ with \#=f or $w$. Furthermore, $\mu_{F K ; A^{w}}^{q, R}(-) \geqslant_{\mathrm{FKG}} \mu_{F K ; A^{f}}^{q, R}(-)$; indeed the finite volume wired measure dominates any other random cluster measure that is defined with the same parameters but other choice of boundary conditions. ${ }^{2}$ In particular, if $A_{1} \subset A_{2}$, then the restriction of $\mu_{F K ; A_{2}^{w}}^{q, R}(-)$ to $A_{1}$ is dominated by $\mu_{F K ;}^{q, R} A_{1}^{w}(-)$ which facilitates passage to the infinite volume limit.

Percolation, in this model, is defined by the limit, as $A \nearrow \mathbb{Z}^{2}$ of the probability that a fixed site is connected to the boundary of $A$ in the wired measure on $A$. If this limit (which is easily shown to exist) is positive, we say there is percolation. When there is no percolation, there is a unique infinite volume limit of $\mu_{F K ; A}^{q, R}(-)$ independent of the finite volume boundary conditions and consequently, for integer $q$, a unique limiting Gibbs state for the corresponding spin-system.

For $A \Subset \mathbb{Z}^{2}$, the dual lattice is defined as follows: For each bond in $\mathbb{B}_{A}$ consider the traversal bond that connects two points of $\left(\mathbb{Z}+\frac{1}{2}\right)^{2}$. The graph consisting of the resulting bonds and their endpoints will be denoted by $\Lambda^{*}$.

\footnotetext{
${ }^{2}$ For integer $q$, all "other boundary conditions" are those that can be obtained via a specification of the spin-state at the boundary. In the random cluster representation, this divides the boundary into components each of which is treated as a single item in the counting of $c(\omega)$ and between which connections are forbidden. For general $q$, these boundary conditions may be directly implemented (with no restriction on the number of separate boundary components) which is more than sufficient for present purposes. As is not hard to see, the restriction of a finite volume measure of this type to a subvolume is a combination of measures of this type for the subvolume.
} 
The measures $\mu_{F K ; A^{*}}^{q_{,}, R}(-)$ induce a measure on the bond configurations of the dual lattice by declaring a dual bond to be occupied/vacant if the corresponding "direct" bond is vacant/occupied. As is well known, these dual measures are also random cluster measures with the same $q$ and bond parameter $R^{*}=q / R$. However, the boundary conditions also transform under duality; in particular, $w \leftrightarrow f$. Ignoring such fine distinctions the selfdual point is therefore just when $R=R^{*}$, i.e., $R=\sqrt{q}$.

In order to investigate a first order transition characterized by a discontinuity in the bond density we must give a precise definition of this object. What is to follow are standard thermodynamic arguments borrowed from the theory of spin systems. We begin with a definition of the free energy:

$$
f \equiv \lim _{\Lambda \times \mathbb{Z}^{2}} \frac{-1}{\beta|\Lambda|} \log Z_{A}^{\#}
$$

where the finite lattices, $A \Subset \mathbb{Z}^{2}$, tends to infinity e.g. in the sense of Van Hove, $Z_{A}^{\#}$ is the partition function with boundary conditions \#, and $|A|$ is the number of sites in $\Lambda$. As is well known, this limit exists independent of the sequence and boundary conditions and $-\beta f$ is convex as a function of $\beta$ or $\log R$. Hence $f$ is differentiable for a.e. $R$, has left and right derivatives for all $R$ and the derivative (with respect to $\log R$ ) is monotone increasing (i.e., non-decreasing).

In finite volume, consider the overall bond density in the \#-state:

$$
n_{A}^{\#}=\frac{1}{|\Lambda|} \frac{\sum_{\omega}|\omega| V^{\#}(\omega)}{Z_{A}^{*}}
$$

Then we claim that at points of continuity of $f^{\prime}$, the limit of $n_{A}^{\#}$ exists and is in fact equal to $-R(d / d R)(\beta f(R))$ - and this defines the bond density. Indeed, writing $R=e^{r}$, then for boundary conditions \# we see that

$$
\frac{Z_{A}^{\#}\left(R=e^{r+\eta}\right)}{Z_{A}^{\#}\left(R=e^{r}\right)}=\left\langle e^{\eta N(\omega)}\right\rangle \underset{F K ; A^{\#}}{q, R=e^{r}} \geqslant e^{\eta\langle N(\omega)\rangle_{F K ; A^{*}}^{q, R}}
$$

(by Jenson's inequality) where $\langle-\rangle \frac{q, R}{F K ; A^{*}}$ denotes the expectation with respect to $\mu_{F K^{\prime} ; A^{*}}^{q, R}(-)$. Taking $A>\mathbb{Z}^{2}$ along any (thermodynamic) sequence for which $n_{A}^{*}$ tend to a definitive limit, we get

$$
-\beta\left[f\left(R=e^{r+\eta}\right)-f\left(R=e^{r}\right)\right] \geqslant \eta n^{\#}\left(R=e^{r}\right)
$$

Thence

$$
-\beta R f_{+}^{\prime}(R) \geqslant n^{\#}(R) \geqslant-\beta R f_{-}^{\prime}(R)
$$


where $f_{ \pm}^{\prime}$ denotes the right and left derivatives (which exist by convexity) respectively. Thus, whenever the derivative of $f$ exists, the overall bond density: $\lim _{A \wedge \mathbb{Z}^{2}}(1 /|\Lambda|)\langle N(\omega)\rangle_{F K ; A}^{q, R} \equiv n(R)$ exists independent of the limiting state.

Next we demonstrate that at points of continuity of $f^{\prime}(R)$, there is a unique state among the translation invariant states and that this state has the appropriate local bond density equal to $\frac{1}{2} n(R)$. (The factor of two is just the bond/site ratio in $d=2$.) Finally, at points of discontinuity, we show that there are (translation invariant) states that achieve the upper and lower density.

First suppose that $R$ is a point of continuity of $n(R)$. Let $\mu_{F K ; a}^{q, R}(-)$ denote any limiting translation invariant state which we suppose has been constructed from finite volume states $\mu_{F K ; A_{k}^{a}}^{q_{1} R}(-)$. Let $\rho^{a}$ denote the bond density in this state; we claim that $2 \rho^{a}(R)=n(R)$. Indeed, let $\Xi_{m}$ denote any thermodynamic sequence of volumes and let $t$ denote the boundary

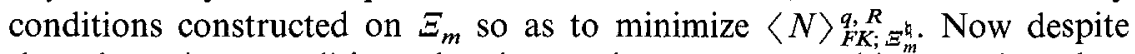
these boundary conditions, by the previous argument this quantity when divided by $\left|\Xi_{m}\right|$ will converge to $n(R)$. Thus, for $m$ large, let us write

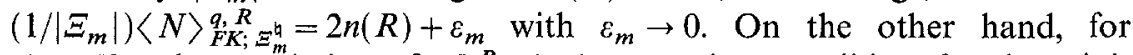
$\Lambda_{k} \supset \Xi_{m}$, the restriction of $\mu_{F K ;}^{q, R} A_{k}^{a}(-)$ to $\Xi_{m}$ is a candidate for the minimizer. For a bond $b$, let $\nabla_{b}(\omega)$ denote the indicator that $b$ is occupied. Then we have $\left.\left(1 /\left|A_{m}\right|\right) \sum_{b \in \mathbb{B}_{A_{m}}}\left\langle\|_{b}(\omega)\right\rangle\right\rangle_{F K}^{q_{1} R} A_{k}^{a} \equiv 2 \rho^{a}+\delta_{k} \geqslant n(R)+\varepsilon_{m}$. Thus, ultimately, $2 \rho^{a} \geqslant n(R)$. Running the same argument where we maximize the bond density in finite volume (here using the wired boundary conditions) we may conclude that $2 \rho^{a} \leqslant n(R)$. Evidently, the bond density is the same in all translation invariant states -in particular equal to the density of the wired state. Now a priori, $\mu_{F K ; w}^{q, R}(-) \geqslant_{F K G} \mu_{F K ; a}^{q, R}(-)$ since this is satisfied in the finite volume $\Lambda_{k}^{w}$ and $A_{k}^{a}$ states. But if the individual bond probabilities are the same, it follows by the corollary to Strassen's theorem (see [L], p. 75) that $\mu_{F K ; w}^{q, R}(-)=\mu_{F K ; a}^{q, R}(-)$. Evidently at points of continuity of $f^{\prime}(R)$ there is but a single translation invariant state.

Finally, if $R_{0}$ is a point of discontinuity we will produce translation invariant states for which the bond densities are exactly $\frac{1}{2} n_{ \pm}\left(R_{0}\right)$. In particular let $A_{k}>\mathbb{Z}^{2}$ and $\eta_{k}$ so small that for any boundary condition \# on $\Lambda_{k}$, the measures $\mu_{F K ; \Lambda^{*}}^{q, R_{0}}-\eta_{k}(-)$ and $\mu_{F K_{0} ; \Lambda^{*}}^{q, R_{0}}(-)$ differ by less than $\vartheta_{k} /\left|\Lambda_{k}\right|$ (in, say, the variational norm) where $\vartheta_{k} \rightarrow 0$. Let $\Xi_{k} \supset \Lambda_{k}$ be so large that (say) the wired measure at $R=R_{0}-\eta_{k}$ on $\Xi_{k}$ restricted to $\Lambda_{k}$ differs from the limiting measure by at most another $\vartheta_{k} /\left|\Lambda_{k}\right|$. Using these restricted measures to provide boundary conditions on $\Lambda_{k}$ but using $R=R_{0}$ we get an approximately translation invariant measure with approximate bond density of $2 n_{-}\left(R_{0}\right)$. Letting $\Lambda_{k}>\mathbb{Z}^{2}$ (along a subsequence if necessary) half the desired result follows. Similar arguments apply to the upper density. 
Thus a discontinuity in the bond density is reflected by the existence of these multiple states with different bond density. Since the absence of percolation in the graphical representation would imply uniqueness of the limiting state, a discontinuity in the bond density is therefore marked by the occurrence of percolation (but not necessarily the onset of percolation).

We have assembled all the necessary ingredients:

Theorem 2.1. The random cluster model on the square lattice has a unique discontinuity in the bond density - unless no discontinuity exists at all. If this discontinuity of the bond density $n(R)$ indeed exists, it occurs precisely at the self-dual point $R=R_{S D}=\sqrt{q}$ and coincides with the magnetic ordering transition/percolation threshold.

Proof. We begin by defining the three relevant bond density parameters: $R_{d}, R_{c}$, and $R_{S D}$. The parameter $R_{d}$ is the bond density parameter at which the purported discontinuity in the bond density occurs, $R_{c}$ is the percolation threshold for the FK representation of the model, and $R_{S D}$ is the self-dual parameter as defined above.

Since, for $q \geqslant 1$, these models with free or wired boundary conditions satisfy all of the necessary conditions, we are able to apply the result of [GKR] concerning the uniqueness of the infinite cluster in two-dimensions. (Although their proof was technically for site models on $\mathbb{Z}^{2}$, the result holds for bond problems as well.) This result excludes the possibility that $R_{c}<R_{S D}$, for the following reason: By the monotonicity of the measure $\left(\mu_{R_{1}}(-) \leqslant_{\mathrm{FKG}} \mu_{R_{2}}(-)\right.$ for $\left.R_{1} \leqslant R_{2}\right)$ there is percolation for all $R>R_{c}$. Thus, from the perspective of the dual model, there is percolation (of dual bonds) for all $R^{*}>R_{c}$ i.e. for all $R<q / R_{c} \equiv R_{c}^{*}$. If $R_{c}$ were below the self-dual point, this would imply an interval, namely $\left(R_{c}, R_{c}^{*}\right)$ at which there would be simultaneous percolation in the direct and dual model. We are thus left with the relation, $R_{c} \geqslant R_{S D}$.

Now by definition, in the region $R<R_{c}$, there is no percolation and hence the (limiting) random cluster measure is unique. A discontinuity in the bond density, however, implies non-uniqueness of the random cluster measure and hence must occur at or above $R_{c}$. Thence, so far, $R_{d} \geqslant R_{c} \geqslant R_{S D}$.

However, it is also not possible to have a discontinuity in the bond density at any $R_{d}$ above $R_{c}$ or $R_{S D}$ because then, by duality, there would be another discontinuity at $R_{d}^{*}=q / R_{d}$-a discontinuity in the density of bonds implies a discontinuity in the density of vacant bonds. We are left with $R_{d}=R_{c}=R_{S D}$. 


\section{THE ASHKIN-TELLER MODEL}

The Ashkin-Teller model [AT] can be described as a four state spin system. At each site $i$ of the lattice $A$ there is a spin $s_{i}$ that may be written in "double Ising form:" $s_{i}=\left(\kappa_{i}, \tau_{i}\right)$, with $\kappa_{i}= \pm 1$ and $\tau_{i}= \pm 1$. In the symmetric case, the Hamiltonian is of the form:

$$
H=-\sum_{\langle i, j\rangle} K\left[\delta_{\kappa, \kappa_{j}}+\delta_{\tau, \tau_{j}}\right]+L\left[\delta_{\kappa_{i} \kappa_{j}} \delta_{\tau_{i} \tau_{j}}\right]
$$

The above Hamiltonian can be generalized to the case where the $\kappa_{i}$ are $r$-state and the $\tau_{i}$ are $s$-state Potts variables. This is the $(r, s)$-cubic model. We will be working in the "orthodox ferromagnetic region" were $K \geqslant 0$ and $L \geqslant 0$ and restrict attention to the case $r=s$. Observe, then that if $L=0$ we have two decoupled $s$-state Potts models while at $K=0$, we have an $s^{2}$-state Potts model.

In the standard FK-type expansion for this model [CM] a bond configuration, $\omega$ is made up of three components: $\omega=\left(\omega_{\kappa}, \omega_{\tau}, \omega_{\kappa \tau}\right)$. The bond configuration $\omega_{\kappa}$ is the configuration of occupied bonds occurring between neighboring sites on the $\kappa$-spin lattice, similarly for $\omega_{\tau}$ while $\omega_{\kappa \tau}$ is the configuration of occupied double bonds each of which have the same effect as two occupied single bonds. Here we will use a slight refinement.

The configurations $\omega$ can be used to define the configurations $\Omega=$ $\left(\Omega_{\kappa}, \Omega_{\tau}\right)$ by the rules $\Omega_{\kappa}=\omega_{\kappa} \vee \omega_{\kappa \tau}$ and $\Omega_{\tau}=\omega_{\tau} \vee \omega_{\kappa \tau}$. Thus these configurations for the $\kappa$ and $\tau$ layers count the presence of either a single or double bond. As derived in [CM] and [PfV], these $\Omega$-configurations are given the graphical weight

$$
W(\Omega)=A^{\left|\Omega_{\kappa} \vee \Omega_{\tau}\right|} B^{\left|\Omega_{\kappa} \wedge \Omega_{\tau}\right|} S^{c\left(\Omega_{\kappa}\right)} S^{c\left(\Omega_{\tau}\right)}
$$

where

$$
A(\beta)=e^{\beta K}-1 \quad \text { and } \quad B(\beta)=\frac{e^{\beta(L+2 K)}-2 e^{\beta K}+1}{e^{\beta K}-1}
$$

Certain properties of the $r \times s$ cubic model in the orthodox region ( $A \leqslant B$ in this formulation) that are essential to our argument were proved in $[\mathrm{CM}]$. There, it was shown that the percolation properties of the graphical representation characterize the phase structure of the spin-system along the same lines as the case of the Potts model. Specifically, there are multiple Gibbs states if there is percolation of the $\Omega_{\kappa}$ or the $\Omega_{\tau}$ bonds and in the absence of either sort of percolation, there is a unique limiting state. Thus, as with the Potts model, when there is a discontinuity in any of the relevant bond densities $\left(\omega_{\kappa}, \omega_{\tau}, \omega_{\kappa, \tau}, \Omega_{\kappa}\right.$ or $\left.\Omega_{\tau}\right)$ there must be $\Omega$-type 
percolation in at least one of the layers. In the symmetric case (but only when $A \leqslant B$ ) it is not hard to show that percolation happens in both layers simultaneously.

We now show that the random cluster measures of the Ashkin-Teller model as defined by the weights in Eq. (9) have various relevant FKG property in the region of interest.

Lemma 3.1. Let $\mu_{A, B}^{s}(-)$ denote the above described random cluster measures associated with symmetric the AT-models. If $s \geqslant 1$ and $B \geqslant A$ then, with the natural partial order on configurations $\Omega$,

(1) The measures $\mu_{A, B}^{s}(-)$ have the strong FKG property and

(2) If $A^{\prime} \geqslant A$ and $B^{\prime} \geqslant B$ then

$$
\mu_{A^{\prime}, B^{\prime}}^{s}(-) \geqslant_{\mathrm{FKG}} \mu_{A, B}^{s}(-)
$$

Proof. We begin with part (1). It is necessary to demonstrate the FKG lattice condition, i.e.,

$$
W\left(\Omega_{1} \vee \Omega_{2}\right) W\left(\Omega_{1} \wedge \Omega_{2}\right) \geqslant W\left(\Omega_{2}\right) W\left(\Omega_{1}\right)
$$

Let us rewrite the weights

$$
W=A^{\left|\Omega_{\kappa}\right|+\left|\Omega_{\tau}\right|}\left[\frac{B}{A}\right]^{\left|\Omega_{\kappa} \wedge \Omega_{\tau}\right|} s^{c\left(\Omega_{\kappa}\right)} s^{c\left(\Omega_{\tau}\right)}
$$

where we used the fact that $\left|\Omega_{\kappa} \vee \Omega_{\tau}\right|+\left|\Omega_{\kappa} \wedge \Omega_{\tau}\right|=\left|\Omega_{\kappa}\right|+\left|\Omega_{\tau}\right|$. We will show that the lattice condition holds for each separate factor.

The factors $s^{c\left(\Omega_{k}\right)}$ and $s^{c\left(\Omega_{\tau}\right)}$ work exactly as in the usual random cluster case, i.e., $c\left(\eta_{1} \vee \eta_{2}\right)+c\left(\eta_{1} \wedge \eta_{2}\right) \geqslant c\left(\eta_{1}\right)+c\left(\eta_{2}\right)$ for any bond configurations $\eta$. Further, for the factor $A^{\left|\Omega_{x}\right|+\left|\Omega_{r}\right|}$, the desired result is an equality. Thus we are down to showing the lattice condition for the quantity $[B / A]^{\left|\Omega_{\kappa} \wedge \Omega_{\tau}\right|}$. To this end, it is sufficient to demonstrate the inequality in the case where the configurations $\Omega_{1} \vee \Omega_{2}$ and $\Omega_{1} \wedge \Omega_{2}$ differ by two bonds. Thus let $a$ and $b$ denote two separate bonds and $\Omega$ a configuration not containing $a$ or $b$. Then we must show

$$
\begin{gathered}
{\left[\frac{B}{A}\right]^{\left|(\Omega \vee a \vee b)_{x} \wedge(\Omega \vee a \vee b)_{\tau}\right|}\left[\frac{B}{A}\right]^{\left|\Omega_{\kappa} \wedge \Omega_{\mathrm{\tau}}\right|}} \\
\geqslant\left[\frac{B}{A}\right]^{\left|(\Omega \vee a)_{\kappa} \wedge(\Omega \vee a)_{\tau}\right|}\left[\frac{B}{A}\right]^{\left|(\Omega \vee b)_{\kappa} \wedge(\Omega \vee b)_{\tau}\right|}
\end{gathered}
$$

or, since $B \geqslant A$, that the sums of the exponents satisfy this inequality. 
Now the bonds $a$ and $b$ can be in one of two situations: they can lie on top of one another, one in each layer, or they can be in separate locations altogether. In the latter case, we have equality. Indeed, suppose that $a$ is in the $\kappa$-layer. Then $\left|(\Omega \vee a)_{\kappa} \wedge \Omega_{\tau}\right|$ increases by one over $\left|\Omega_{\kappa} \wedge \Omega_{\tau}\right|$ if (and only if) $\Omega$ has a matching bond for $a$ in the $\tau$-layer. But in this case, $\left|(\Omega \vee a \vee b)_{\kappa} \wedge(\Omega \vee b)_{\tau}\right|$ has also increased by one over $\mid(\Omega \vee b)_{\kappa} \wedge$ $(\Omega \vee b)_{\tau} \mid$. Furthermore, if there in no matching bond for $b$ in $\Omega$ then $\left|(\Omega \vee a \vee b)_{\kappa} \wedge(\Omega \vee b)_{\tau}\right|=\left|(\Omega \vee b)_{\kappa} \wedge(\Omega \vee b)_{\tau}\right|$ and if $b o t h a$ and $b$ have matching bonds then $\left|(\Omega \vee a \vee b)_{\kappa} \wedge(\Omega \vee a \vee b)_{\tau}\right|=\left|\Omega_{\kappa} \wedge \Omega_{\tau}\right|+2$. Thus, we are down to the case where $a$ and $b$ are in the same location. But here, $\left|(\Omega \vee a \vee b)_{\kappa} \wedge(\Omega \vee a \vee b)_{\tau}\right|=\left|\Omega_{\kappa} \wedge \Omega_{\tau}\right|+1$ while $\left|(\Omega \vee a)_{\kappa} \wedge(\Omega \vee a)_{\tau}\right|$ and $\left|(\Omega \vee b)_{\kappa} \wedge(\Omega \vee b)_{\tau}\right|$ both equal $\left|\Omega_{\kappa} \wedge \Omega_{\tau}\right|$.

For the proof of part (2) we simply write the primed weight as the unprimed weight augmented by a function:

$$
\mu_{A^{\prime}, B^{\prime}}^{s}(\Omega) \propto\left[\frac{A^{\prime}}{A}\right]^{\left|\Omega_{\kappa} \vee \Omega_{\tau}\right|}\left[\frac{B^{\prime}}{B}\right]^{\left|\Omega_{\kappa} \wedge \Omega_{\tau}\right|} \mu_{A, B}^{s}(\Omega)
$$

Since this augmenting function is manifestly an increasing function the result is apparent.

The duality relations for these graphical representations were established in [CM] and [PfV] generalizing the result of [DR]. In summary, for a given configuration on the regular lattice, the dual configuration is constructed by occupied bonds of $\Omega_{\kappa}$ and $\Omega_{\tau}$ representing vacant $\Omega_{\kappa}^{*}$ and $\Omega_{\kappa}^{*}$ bonds on the dual lattice. The dual parameters, $A^{*}$ and $B^{*}$ are found to be given by the relations $A^{*}=s B^{-1}$, and $B^{*}=s A^{-1}$. By equating $W_{A, B}$ to $W_{A^{*}, B^{*}}^{*}$, the self dual line is found to be $A B=s$.

Theorem 3.2. For the symmetric Ashkin-Teller model in the region $A \leqslant B$ (orthodox ferromagnetic region) or the associated random cluster measures (with $s \geqslant 1$ ) any discontinuity in the $\Omega_{\kappa}$ or $\Omega_{\tau}$ bond density must occur on the self-dual curve $A B=s$. Furthermore, should such a discontinuity occur, both bond densities are discontinuous and this is accompanied by the onset of spontaneous magnetization or percolation.

Proof. The argument follows closely the one for the case of the Potts model. Since, for $A \leqslant B$ and $s \geqslant 1$ the measures $\mu_{A, B}^{s}(-)$ (defined by limiting processes with appropriate boundary conditions) are FKG, etc. so are the $\Omega_{\kappa}$ and $\Omega_{\tau}$ marginal. We may apply the [GKR] theorem to these objects. Suppose that at the point $(\bar{A}, \bar{B})$ with $\bar{A}=\lambda \bar{B}, \lambda \leqslant 1$ with there is discontinuity. Explicitly, there are two states where (say) the $\Omega_{\kappa}$ bond densities are different. Consider the trajectory $A=\lambda B, 0 \leqslant B<\infty$ that passes 
through this point. It is observed that the dual model follows the same trajectory (in the opposite direction). It is also noted that as $A$ and $B$ increase along the trajectory so do the measures (in the sense of FKG). Hence the argument for the Potts model may be applied directly and we conclude that this is the unique point on the trajectory where the $\Omega_{\kappa}$ bond density is discontinuous and it is also the threshold for percolation in the $\kappa$ layer. (And also that $\bar{A}=\sqrt{s \lambda}$ and $\bar{B}=\sqrt{s / \lambda}$.) Finally, in the wired state (which is symmetric between layers) if $A<\bar{A}$ and $B<\bar{B}$ there is no percolation in either layer while if $A>\bar{A}$ and $B>\bar{B}$ there is percolation in both layers and thus, by the FKG property simultaneous percolation in both layers.

\section{DISCONTINUOUS TRANSITIONS IN THE AT MODEL}

In this final section, we will demonstrate that for (integer) $s$ sufficiently large, there are always discontinuous ordering transitions in the AT models we have studied. In particular, along every trajectory $A=\lambda B$ with $\lambda \in(0, \infty)$ fixed and $B: 0 \rightarrow \infty$ there is a discontinuous phase transition. Hence, for $\lambda \leqslant 1$ the ordering transition is unique and occurs precisely on the self dual line. However, the situation when $B<A$ may be very different; for example we will show that if $B \ll A$, there are (at least) two ordering transitions.

The method we use is the standard "large entropy" argument that was introduced in [KS]. The proof will be simplified by the recent observation [BCK], [CM] that graphical representations of the type used here are themselves reflection positive. Our starting point is a Theorem from [KS].

Lemma 4.1. Let $a$ and $b$ denote two distinctive of a bond. Let $H$ be a Hamiltonian that depends on a control parameter, denoted by $\alpha$, that lies in the range $\left[\alpha_{a}, \alpha_{b}\right]$ and let $\langle-\rangle_{N, \alpha}$ denote the Gibbs state on the torus $\mathscr{T}_{N}$ of $N$ sites induced by the Hamiltonian $H$ at parameter value $\alpha$. Finally let $c_{1} \in\left(\frac{1}{2}, 1\right]$ and $c_{2} \in[0,1]$ be such that $c_{2} \leqslant\left[\frac{1}{2}+\sqrt{\frac{1}{2}-\left(c_{1} / 2\right)}\right]^{2}$ and let $\varepsilon_{a}, \varepsilon_{b} \in\left(0, \frac{1}{2}\right)$. Suppose that for all $\alpha \in\left[\alpha_{a}, \alpha_{b}\right]$, and for all bonds $c, \tilde{c} \in \mathscr{T}_{N}$, one has

(0) $\chi_{a}(c) \chi_{b}(c)=0$,

(i) $\left\langle\chi_{a}(c)+\chi_{b}(c)\right\rangle_{N, \alpha} \geqslant c_{1}$,

(ii) $\left\langle\chi_{a}(c) \chi_{b}(\tilde{c})\right\rangle_{N, \alpha} \leqslant c_{2}$,

and, meanwhile,

(iiia) $\left\langle\chi_{a}(c)\right\rangle_{N, \alpha_{a}}>1-\varepsilon_{a}$

and

(iiib) $\left\langle\chi_{b}(c)\right\rangle_{N, \alpha_{b}}>1-\varepsilon_{b}$. 
where in the above, $\chi_{b}(c)$ denotes the event that the bond $c$ is in the state $a$, etc. Furthermore, suppose that the above holds for all $N$ in some sequence $\mathscr{T}_{N} \nearrow \mathbb{Z}^{d}$. Then there is a value $\alpha_{t} \in\left(\alpha_{a}, \alpha_{b}\right)$ and two distinct (infinite volume) states $\langle-\rangle_{\alpha_{t}}^{a}$ and $\langle-\rangle_{\alpha_{1}}^{b}$ (characterized, e.g., by the fact that $\left\langle\chi_{a}(c)\right\rangle_{\alpha_{t}}^{a} \geqslant 1-\delta$ and $\left\langle\chi_{b}(c)\right\rangle_{\alpha_{t}}^{b} \geqslant 1-\delta$, where $\delta$ is a particular function of $c_{1}$ and $c_{2}$ such that $\delta \rightarrow 0$ as $c_{1} \rightarrow 1$ and $c_{2} \rightarrow 0$ ).

Proof. See e.g. [KS] or [BCK].

As was discussed in the latter reference, it is not hard to show that the above also applies to graphical representations for spin-systems that are of the type used here. For present purposes, it is sufficient to note that the bond variables may be incorporated into a genuine Gibbsian structure by the construction of an equivalent annealed bond-diluted model.

In what is to follow, we will restrict attention to $d=2$ which, in particular allows us to take advantage of the diagonal torus (SST). However, the results that follow concerning discontinuous transitions and intermediate phases can readily be extended to higher dimensions.

Our bond events will be: (a) that at least one of $\Omega_{\tau}(c)$ or $\Omega_{\kappa}(c)$ is occupied and (b) that they are both vacant. It is clear that (0), (i) and (iii) are satisfied. We now show:

Lemma 4.2. For the $s \times s$ AT models and their associated graphical representations in the region of parameters where $A \geqslant 0$ and $B \geqslant 0$ if $s$ is sufficiently large then $\left\langle\chi_{a}(c) \chi_{b}(\tilde{c})\right\rangle_{N ; A, B}$ is uniformly small.

Proof. Using the standard chessboard arguments, it is sufficient to show that a "contour element" has small probability. In our case, a contour element is a site that is the endpoint of one bond of the $a$-type and another of the $b$-type. However at any such site, one bond of each type meet at right angles, thus it is sufficient to consider the case where $c$ and $\tilde{c}$ meet at the origin and point down the coordinate axes. Let us write

$$
\chi_{a}(c)=\chi_{f}(c)+\chi_{\tau}(c)+\chi_{\kappa}(c)
$$

where $f$ is the event that both bonds are present, $\tau$ is the event that the $\tau$-bond is present but not the $\kappa$-bond, etc. We will separately estimate $\left\langle\chi_{f}(c) \chi_{b}(\tilde{c})\right\rangle_{N ; A, B},\left\langle\chi_{\tau}(c) \chi_{b}(\tilde{c})\right\rangle_{N ; A, B}$ and $\left\langle\chi_{\kappa}(c) \chi_{b}(\tilde{c})\right\rangle_{N ; A, B}$. Applying multiple reflections until the torus is covered, we arrive at

$$
\left\langle\chi_{f}(c) \chi_{b}(\tilde{c})\right\rangle_{N ; A, B} \leqslant\left[Z_{N ; f: b} / Z_{N}\right]^{1 / N}
$$

where $Z_{N}=Z_{N}(A, B)$ is the partition function for the torus $\mathscr{T}_{N}$ and $Z_{N ; f: b}$ is the constrained partition function given that the bonds of $f$ and $b$-type 
form the alternating (diagonal) striped pattern which results from the multiple reflections: All the even sites are contour sites while the odd diagonal stripes alternate between stripes of sites emanating $f$-bonds and stripes of sites emanating vacant bonds. (See e.g., $[\mathrm{S}]$ or $[\mathrm{CM}]$ Section IV for a more detailed version of an argument of this type.) Similar estimates are obtained for $\left\langle\chi_{\tau}(c) \chi_{b}(\tilde{c})\right\rangle_{N ; A, B}$ and $\left\langle\chi_{\kappa}(c) \chi_{b}(\tilde{c})\right\rangle_{N ; A, B}$ with the relevant constrained partition functions denoted by $Z_{N ; \tau: b}$ and $Z_{N ; k: b}$ respectively.

The constrained partition functions are easily calculated:

$$
\lim _{N \rightarrow \infty}\left[Z_{N ; f: b}\right]^{1 / N}=A B s^{1 / 2}
$$

and

$$
\lim _{N \rightarrow \infty}\left[Z_{N ; \tau: b}\right]^{1 / N}=\lim _{N \rightarrow \infty}\left[Z_{N ; \kappa: b}\right]^{1 / N}=s^{5 / 4} A
$$

We may estimate

$$
Z_{N} \geqslant(A B)^{2 N}+s^{2 N}+A^{2 N} s^{N}
$$

All of the required estimates work exactly as in the Potts model by selecting the two appropriate terms from Eq. (17). Thus

$$
\lim _{N \rightarrow \infty}\left\langle\chi_{\tau}(c) \chi_{b}(\tilde{c})\right\rangle_{N ; A, B} \leqslant \lim _{N \rightarrow \infty} \frac{s^{5 / 4} A}{Z_{N}^{1 / N}} \leqslant \frac{s^{1 / 4} A}{\left[s^{N}+A^{2 N}\right]^{1 / N}} \leqslant s^{-1 / 4}
$$

Similarly,

$$
\lim _{N \rightarrow \infty}\left\langle\chi_{f}(c) \chi_{b}(\tilde{c})\right\rangle_{N ; A, B} \leqslant \lim _{N \rightarrow \infty} \frac{A B s^{1 / 2}}{\left[(A B)^{2 N}+s^{2 N}\right]^{1 / N}} \leqslant s^{-1 / 4}
$$

These are indeed small if $s$ is large.

Theorem 4.3. Consider the $s \times s$ AT model with $s$ large. Then, if $0<A \leqslant B$ there is a discontinue phase transition at every point of the selfdual line $A B=s$ and everywhere else the (energy) density is continuous. If $A>B$, there are also discontinuous transitions separating the high and low temperature phases however, these need not take place on the self-dual curve. In particular, along the trajectories $B=\varepsilon A$ if $\varepsilon$ is sufficiently small there are two phase transitions and the immediate vicinity of the self-dual curve is an intermediate phase.

Proof. The consequence of Lemma 4.2 is that (for large $s$ ) if we move along the trajectory $A=\lambda B$ then at some point we will hit a discontinuity 
in some bond density. (For these systems, bond density and energy density are easily related. $\mathrm{Cf}$., [CM] footnote 4.) If $\lambda<1$, then, according to Theorem 3.2, this is the unique point of discontinuity along this trajectory. Hence the entire self-dual curve is a curve of phase coexistence and is the boundary between the ordered and disordered phases.

Now consider the case $B=\varepsilon A, \varepsilon \ll 1$ with $0<A<\infty$. Obviously if $A$ is sufficiently large/small then both layers are nearly full/empty. However, let us consider an intermediate value of the parameter namely the self-dual point $A=A^{*}(\varepsilon)=(s / \varepsilon)^{1 / 2}$. We see

$$
\lim _{N \rightarrow \infty}\left\langle\chi_{f}(c)\right\rangle_{N ; A^{*}(\varepsilon), \varepsilon A} \leqslant \frac{\left(A^{*} B^{*}\right)^{2}}{\left(A^{*}\right)^{2} S} \leqslant \varepsilon
$$

and

$$
\lim _{N \rightarrow \infty}\langle\chi \varnothing(c)\rangle_{N ; A^{*}(e), \varepsilon A} \leqslant \frac{s^{2}}{\left(A^{*}\right)^{2} S} \leqslant \varepsilon
$$

Thus at the self dual point (and, by continuity of these estimates in its immediate vicinity) these objects are rare. However $\tau$ - and $\kappa$-bonds do not easily coexist for any value of $A$. Indeed

$$
\lim _{N \rightarrow \infty}\left\langle\chi_{\kappa}(c) \chi_{\tau}(\tilde{c})\right\rangle_{N ; A, B} \leqslant \frac{A^{2} s^{1 / 2}}{A^{2} s} \leqslant s^{-1 / 2}
$$

Thus, in the vicinity of the self-dual point there are at least two (or more precisely, at least $2 \mathrm{~s}$ ) phases. In one of these, the $\kappa$ layer is nearly full at the expense of a nearly vacant $\tau$-layer and vise versa for the other phase. Evidently, in this region of the phase diagram there is an intermediate phase and (at least) two phase transitions along trajectories of the type described.

Remark. Using the above techniques it is not difficult to show that there are in fact two discontinuous transitions along these trajectories.

\section{REFERENCES}

[A] K. S. Alexander, On weak mixing in lattice models, Preprint (1996).

[ACCN] M. Aizenman, J. T. Chayes, L. Chayes, and C. M. Newman, Discontinuity of the magnetization in one-dimensional $1 /|x-y|^{2}$ Ising and Potts models, J. Stat. Phys. 50:1-40 (1988).

[AT] J. Ashkin and E. Teller, Statistics of two-dimensional lattices with four components, Phys. Rev. 64:178-184 (1943). 
[BCK ] M. Biskup, L. Chayes, and R. Kotecký, Coexistence of partially disordered/ ordered phases in an extended Potts model, Preprint (1997).

[CM] L. Chayes and J. Machta, Graphical representations and cluster algorithms. Part I: Discrete spin systems, Physica A 239:542-601 (1997).

[DR] E. Domany and E. Riedel, Two-dimensional anisotropic $N$-vector models, Phys. Rev. B 19:5817-5834 (1979).

[FK] C. M. Fortuin and P. W. Kasteleyn, On the random cluster model. I. Introduction and relation to other models, Physica 57:536-564 (1972).

[G] G. Grimmett, The stochastic random-cluster process and the uniqueness of random-cluster measures, Ann. Probab. 23:1461-1510 (1995).

[GKR ] A. Gandolfi, M. Keane, and L. Russo, On the uniqueness of the infinite occupied cluster in dependent two-dimensional site percolation, Ann. Prob. 16:1147-1157 (1988).

[KS] R. Kotecký and S. B. Shlosman, First-order phase transitions in large entropy lattice models, Comm. Math. Phys. 83:493-515 (1982).

[L] T. M. Liggett, Interacting Particle Systems (Springer Verlag, Heidelberg, New York, 1985).

[LMMsRS] L. Laanait, A. Messager, S. Miracle-Solé, J. Ruiz, and S. Shlosman, Interfaces in the Potts model. I: Pirogov-Sinai theory of the Fortuin-Kasteleyn representation, Comm. Math. Phys. 140:81-91 (1991).

[PfV] C. E. Pfister and Y. Velenik, Random-cluster representation of the AshkinTeller model, J. Stat. Phys. 88:1295-1331 (1997). 